disciplines were consulted. They were asked whether an information leaflet would be beneficial as a prompt to offer parents hospice care once the acute hospital services are no longer required.

Intervention A leaflet was designed by collaborating with staff at the neonatal unit, the hospice (both clinical, family support and bereavement team) and parents of children who have utilised the services of both the neonatal unit and the hospice.

The leaflet explains the services available for transitional care using case studies of babies who have successfully been discharged home having stayed in the hospice for a period of time.

As well as informing parents, the leaflet was designed to act as a prompt for neonatal staff to inform parents that the hospice can provide transitional and ongoing care for their child if they wished to attend.

Study design Semi structured interviews with staff on the neonatal unit and the hospice setting

Semi structured interviews with parents on the neonatal unit and the hospice setting

Strategy for change Implementation was provided by sending correspondence to members of the neonatal team informing them that the leaflet had been created and was available for use. The palliative care team from the hospice introduced this at the monthly neonatal multidisciplinary team meetings and the grandround.

This project was introduced over a 6 month period.

Measurement of improvement Prospectively an increased number of children were referred following the implementation of the leaflet. Very positive parental feedback was received.

Effects of changes Significantly more babies with life limiting conditions and ongoing complex needs were referred to the hospice team for either transitional care or ongoing support.

Parents of babies eligible for the service were given wider patient choice about attendance.

The only problem encountered was the perception by parents and staff that a hospice can only provide support for babies that are imminently dying. The aim of the leaflet was to alter that perception.

Lessons learnt This work taught us the importance of collaborating with a muiltidisciplinary team when introducing a new resource in order to make it successful.

Message for others That hospices provide an excellent place for the interim care of life limited babies with ongoing complex needs. They can be looked after by their parents with the support of specialist palliative care services during their transition from the acute hospital setting to home.

The leaflet is a useful source of information for neonatal staff informing parents of the services available in their local hospice.

'Together for Short Lives' have shown an interest in this project.

\section{G572(P) INDIVIDUALISED CARE ROOMS: THE FUTURE OF NEONATAL CARE}

E Llew, C Cane. Starlight Neonatal Unit, Barnet Hospital, Royal Free London NHS Foundation Trust, London, UK

10.1136/archdischild-2015-308599.521

Context Individualised care rooms (ICR) were established in District General Hospital with a Level 2 Neonatal Unit. This was a result of consultation between the medical health professionals, members of the allied health professionals and parent groups.

Problem In a traditional Neonatal Intensive Care (NICU) setting, the majority of care is undertaken by neonatal nurses and incubators are set out in an 'open bay' layout.

Assessment of problem and analysis of its causes As a result of this traditional setting, parents often report feeling disempowered. The lack of privacy also limits opportunities for kangaroo care and this can affect parent-child bonding.

In the Individualised Care Rooms (ICR) model, parents are encouraged to be resident alongside their premature baby from an early stage and become integral to their daily care in a developmental care environment. They are supported by doctors and nurses, and the babies are monitored by cardiac monitoring and pulse oximetry.

Research has shown that there is greater parents' satisfaction with the ICR model and premature babies benefit from a shorter length of stay and lower incidence of bronchopulmonary dysplasia. Evidence also supports the provision of regular kangaroo care to improve cerebral function.

Intervention There are three broad phases:

First phase: Design and building of the ICR. We converted an area within our neonatal unit that was not in clinical use into 10 fully equipped individual rooms. Each room is designed specifically to allow adequate natural lighting, ventilation and space for an adult bed and an incubator. The rooms are also equipped with monitoring equipment and amenities including washbasin and television. Parents share bathrooms and dayroom facilities. A nursing station with central monitor is manned by trained neontal nurses to support parents and babies.

Second phase: Education and training. We formulated an admission policy and expected standard of care for the ICR. We set off to promote the ICR in a launch event. There is an ongoing programme of education and training sessions for health professionals and parent groups.

Third phase: Admission of babies to the ICR since December 2013.

Study design We are in the process of designing a pilot cohort analytical study comparing outcomes of premature babies in the ICR with matched controls of babies nursed in the open bay in our neonatal unit. Our primary outcome targets will include duration of stay, gestational age at discharge and weight at discharge. The secondary outcome target will be a formal neurodevelopmental assessment at various corrected gestation age timepoints.

Strategy for change The implementation of the ICR was carried out in a phased manner. This allows adequate time for specific consideration in the design and build of the rooms, education and training sessions, troubleshooting and lastly, implementation. Measurement of improvement Alongside with the planned pilot cohot analytical study, we also plan to conduct a parent satisfaction survey to obtain formal feedback about the ICR.

Effects of changes Observation from health professionals are that babies are being discharged at a younger gestational age and lower birth weight with no significant unintended consequence in the community. Verbal feedback from the parent groups regarding ICR has been overwhelmingly positive so far. However we hope to produce a more formal and measurable outcome with the planned study and parents' satisfaction survey.

Lessons learnt With the change of junior doctors every six months, education and training sessions need to be ongoing to promote awareness of the purpose and benefits of ICR. Ongoing troubleshooting sessions also help fine tune the running of ICR. 
Message for others Based on current evidence, we believe that the ICR model should be taken into consideration in the future design and planning of any neonatal unit.

\section{G573(P) IMPROVING EASE AND ACCURACY OF CENTILE CALCULATIONS WITH A SMARTPHONE IMPLEMENTATION OF RCPCH UK-WHO GROWTH CHARTS}

CJ Kelly. Centre for the Developing Brain, King's College London, London, UK

10.1136/archdischild-2015-308599.522

Context Improvement work performed while working as a paediatric trainee ST2 on a tertiary neonatal intensive care unit, neurosurgical ward and academic research department. Target audience: health professionals who calculate growth centiles on a regular basis.

Problem Calculating growth centiles and their trends are a crucial part of the general paediatric and neonatal assessment. However, due to the regular scarcity of paper charts and inconvenience of trying to find one on a busy ward, centiles are often overlooked. Even with a chart available for use, the manual plotting method gives broad estimates rather than exact numbers (i.e. $25-50^{\text {th }}$ centile), which are not appropriate for research purposes.

Assessment of problem and analysis of its causes Working on a paediatric neurosurgical ward, the neurosurgical consultants complained that growth centiles were not being filled in consistently by junior doctors during clerking. A poll of fellow trainees agreed that an electronic version, available at the point of care, would be very beneficial. Problems with paper growth charts include lack of chart availability, time pressure, plotting inaccuracies, and inherent centile approximation due to threshold banding. Additionally, the calculation of corrected age for children and post menstrual ages for premature babies is commonly performed inaccurately.

A solution to this problem would need to combine improved accuracy with greater ease of calculation, providing a tool that staff could carry with them everywhere at work.

Intervention Creation of a smartphone app to calculate growth centiles for premature babies through to 18 year old children, using RCPCH UK-WHO data. The app also addresses the difficulty of calculating corrected ages by building this into the calculation.

Strategy for change The provisional app was circulated to a number of interested paediatricians, and very helpful feedback received from the NHS Hackday group. The app was also discussed with Prof Tim Cole (the creator of the original growth charts), who provided some very helpful feedback and suggestions for improvement.

The app was launched as a free download in October 2014 on iPhone and Android as a proof of concept via social media, and a significant amount of constructive feedback was received. An updated version is currently in progress, building in many of these suggestions.

Measurement of improvement The project is at an early stage, and no objective metrics of improvement have yet been collected. It is hoped that this will be possible after the launch of the final version.

\section{GrowthCharts $^{\text {ukmomo }}$}

\section{Current gestational age: $40+2$ weeks, day 7 today*}

\section{RCPCH UK-WHO Growth Chart for Boys}

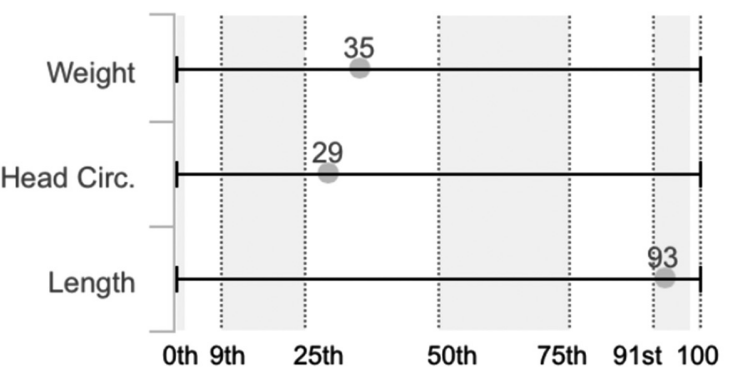

\section{Weight $3.5 \mathrm{~kg} 25$ th - 50th centile (35th) \\ Head $35 \mathrm{~cm} 25$ th -50 th centile (29th) \\ Length $55 \mathrm{~cm} 91 \mathrm{st}$ - 98th centile (93rd)}

\section{Measured on Sun 7th Sep 2014}

\section{Modify Details}

\section{New Chart}

\section{Source information and Disclaimer}

Abstract G573(P) Figure 1

Effects of changes The app is now being used in the research department at St Thomas' to calculate accurate centiles for research participants at the Centre for the Developing Brain. Despite the deliberately local launch of the first version, the app has already been downloaded by 2,500 users. It is hoped that as the app gains adoption, the calculation of growth centiles in paediatric practice will become more widespread, with associated benefits to child health (Figure 1).

Lessons learnt I have learned about implementing a new tool in clinical practice, combined with gathering feedback and suggestions from a wide range of people to make the product as clinically useful as possible.

Message for others Creating a web/app-based tool for commonly-performed error-prone tasks appears to be an effective strategy to improve quality at the bedside. There are many resources available online to guide interested people through this process. 